\title{
Retinal Biomarkers of Alzheimer's Disease: Insights from Transgenic Mouse Models
}

\author{
Rui Bernardes ${ }^{1,2 *}$, Gilberto Silva ${ }^{1}$, Samuel Chiquita ${ }^{1}$, Pedro Serranho ${ }^{1,2,3}$, and \\ António Francisco Ambrósio ${ }^{1}$ \\ 1 Institute for Biomedical Imaging and Life Sciences (IBILI), Faculty of Medicine, \\ University of Coimbra, Coimbra, Portugal \\ ${ }^{2}$ Coimbra Institute for Biomedical Imaging and Translational Research (CIBIT), \\ ICNAS, University of Coimbra, Coimbra, Portugal \\ 3 Department of Sciences and Technology, Universidade Aberta, Lisboa, Portugal \\ *rmbernardes@fmed.uc.pt
}

\begin{abstract}
In this paper, we use the retina as a window into the central nervous system and in particular to assess changes in the retinal tissue associated with the Alzheimer's disease. We imaged the retina of wild type (WT) and transgenic mouse model (TMM) of Alzheimer's disease with optical coherence tomography and classify retinas into the WT and TMM groups using support vector machines with the radial basis function kernel. Predictions reached an accuracy over $80 \%$ at the age of 4 months and over $90 \%$ at the age of 8 months. Texture analysis of computed fundus reference images suggest a more heterogeneous organization of the retina in transgenic mice at the age of 8 months in comparison to controls.
\end{abstract}

Keywords: Alzheimer's Disease, 3xTg Mouse Model, Optical Coherence Tomography, Retina, Classification

\section{Introduction}

In this work we explore the use of statistical descriptors of Optical Coherence Tomography (OCT) data of wild type (WT) and transgenic mouse model (TMM) of Alzheimer's Disease (AD). Our objective is two-fold. On the one hand, we aim to test the classification approach previously applied to OCT data of human retinas to classify those into the healthy control and disease groups [1. On the other hand, we aim to clarify if changes in the retina are present and can be detected in the early stages of disease.

According to the WHO (World Health Organization) report [2] projections from 2013 estimate a total of over 47 million people living with dementia (including AD) in 2015. Projections for 2030 are of 66 million, resulting in the indisputable burden for patients and caregivers. Although these figures account for all types of dementia, AD is the most common type and estimated to represent $60 \%$ to $80 \%$ of the cases [3]. 
One of the fundamental problems tackling AD is the lack of early diagnostic biomarkers, the reason why AD may develop undiagnosed for over a decade 4 . While the definite diagnosis of AD can only be "confirmed after a postmortem examination of the brain" 4, up to now the "probable" diagnosis is possible only when "significant neurological damage has already occurred" 4].

A particular effective diagnostic tool requires positron emission tomography (PET) imaging facilities and the use of the Pittsburgh compound-B (PiB) agent, therefore severely limiting the access to this biomarker due to the (high) associated costs and due to the limited number of PET facilities [4].

Ideally, a biomarker should be easy to collect and measure in addition to the objective and reproducible mandatory characteristics [5. The definition of biomarker clearly points to the need of a different approach when it comes to AD diagnosis. The use of the retina as a window into the brain is being followed by an increasing number of research groups and appears as the natural choice. The retina is actually the visible part of the central nervous system (CNS). There is also cumulating evidence indicating that the retina can be also affected in neurodegenerative diseases [6].

OCT is a wide spread imaging technique in the field of ophthalmology and is an important tool to diagnose a variety of diseases. OCT provides the absorption and scattering properties of the tissues and thus inherently conveys pertinent information on the structure of the retina. In addition to the basic information on the thickness of the retina, previous studies conducted by our research group demonstrate that information gathered by the OCT allows to automatically classify eyes into the Parkinson, Diabetic Retinopathy and Multiple Sclerosis groups [78. These works paved the way for the work herewith reported.

\section{Methodology}

The use of transgenic mouse models of AD allows to overcome a fundamental problem when trying to collect data in the early stages of the disease in humans, that the diagnosis of $\mathrm{AD}$ is possible only after a significant neurological damage occurred 4. Furthermore, it allows to follow-up the natural development and ageing of the retina for WT controls and TMM and to compare the two groups at the same age at regular intervals during their life-span.

The TMM used in this study is the $3 \mathrm{xTg}-\mathrm{AD}$ (triple-transgenic mouse model). This model presents characteristics of the human form of disease, namely the aggregation of amyloid- $\beta(\mathrm{A} \beta)$ protein accumulating into plaques and the hyperphosphorylation of tau protein 9 .

In this work we report data gathered at the ages of 4 and 8 months.

\section{$2.1 \quad$ OCT Imaging}

OCT is a noninvasive diagnostic imaging technology able to provide crosssectional images (B-scans) of the retinal tissue in vivo and in situ (Fig. 11). Its working principle is analogous to that of ultrasonography with light taking the 
role of sound [10. Furthermore, OCT terms such as A-scan and B-scan match the definition of those in ultrasound. The principle, based on the backscattering of low-coherence light has been extensively described in the literature 1112]. OCT readings convey information on refractive index changes along the light path. Consequently, any change in the content or structural organization of the retina, with respect to the healthy condition, is captured in the statistics of the signal.

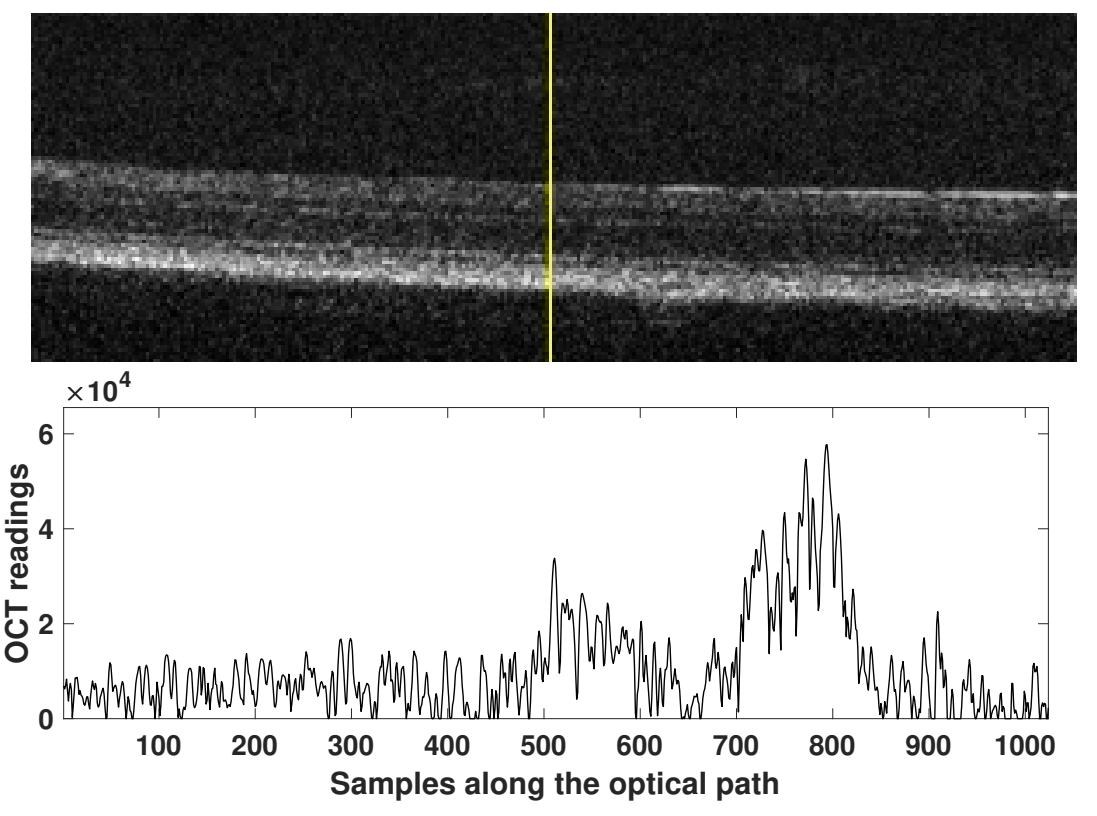

Fig. 1. Optical coherence tomography. Top: B-scan of the right eye of a wild type (control) mice at the age of 8 months. Bottom: Plot of the A-scan earmarked in yellow (B-scan above). A-scan values up to sample $\sim 500$ correspond to OCT readings within the vitreous. A-scan values from sample $\sim 500$ to $\sim 800$ correspond to OCT readings within the retina and A-scan values from sample $\sim 800$ to 1024 correspond to OCT readings within the choroid. Light travels from top to bottom (B-scan) and left to right (A-scan).

The system used in this study is the Micron IV OCT (Phoenix Research Labs, Pleasanton, CA, USA). It is tailored to image mouse and rat retinas and allows to scan the posterior pole of the eye gathering 512 by 512 by 1024 voxels over $50^{\circ}$ field of view.

The animals were anesthetized and pupils dilated. Animals were then placed in front of the OCT for data acquisition with all scans being performed at the 
same retinal location, horizontally centered on the optic disk and vertically above the optic disc border.

A total of 512 B-scans, of 512 A-scans each, were produced per eye scan. Each B-scan is saved in the computer disc as an uncompressed TIFF file of 512 by 1024 pixels. The 512 images were then read using Matlab 9.1.0 (R2016b) (The MathWorks Inc., Natick, MA, USA) for processing and analysis.

\section{$2.2 \quad$ Pre-Processing}

OCT data was saved in a format read by the OCT Explorer (Retinal Image Analysis Lab, Iowa Institute for Biomedical Imaging, Iowa City, IA) 1314 115 software for segmentation. In particular we take advantage of the ease to perform manual segmentation/correction using this software interface to segment three major layers. The first major layer (Layer 1) comprises the retinal nerve fiber layer (RNFL), the ganglion cell layer (GCL), the inner plexiform layer (IPL), the inner nuclear layer (INL) and the outer plexiform layer (OPL) layers. The second major layer (Layer 2) comprises the outer nuclear layer (ONL) and the external limiting membrane (ELM), and; the third major layer (Layer 3) comprises the ellipsoid zone and retinal pigment epithelium (RPE) layers. For a detailed insight into the histology, OCT images and schematic arrangement of the mouse retina, please refer to [4]16 17]18 19].

\section{$2.3 \quad$ Processing}

Histograms Histograms contain the first-order statistical information about the images/volumes. These are an obvious choice when looking for differences in the very early stages of disease, when the structure of the retina is still preserved and indistinguishable from the healthy condition. Characteristics of the imaged tissue (retina) can thus be obtained by analyzing the histograms of the OCT values. Histograms were computed and the respective probability mass function $(p m f)$ determined separately for Layer $1(j=1)$, Layer $2(j=2)$, Layer $3(j=3)$ and the whole retina $(j=4)$.

Parameters defining the shape of the pmf are then computed by fitting the function defined by (1)

$$
f(x)=\sum_{i=0}^{2} A_{i} e^{-\left(x-x_{i}\right)^{2} /\left(\sigma_{i}^{2}\right)}
$$

to the pmf, from where a vector of parameters $\left(\mathbf{v}_{j}=\left[A_{0}, x_{0}, \sigma_{0}, A_{1}, x_{1}, \sigma_{1}\right.\right.$, $\left.\left.A_{2}, x_{2}, \sigma_{2}\right]\right)(j=\{1,2,3,4\})$ is computed conveying information on the characteristics of the tissue.

Each of the four individual vectors $\left(\mathbf{v}_{j}\right)$ is transformed into the vector $\mathbf{w}_{j}=\left[A_{0}, \sigma_{0}, A_{1}, \sigma_{1}, A_{2}, \sigma_{2}, x_{0}-x_{1}, x_{0}-x_{2}, A_{0} / A_{1}, A_{0} / A_{2}, \sigma_{0} / \sigma_{1}, \sigma_{0} / \sigma_{2}, \mathrm{RMSE}\right]$, where RMSE stands for the root mean squared error of the fit. 
Mean value fundus images Different approaches can be used to compute a fundus reference image from an OCT volume data. These approaches share the idea of using depth-wise averaging (total or partial) of each individual Ascan 20. In this work, for each of the three layers (Layer 1, Layer 2 and Layer 3 ) a mean value fundus (MVF) image [21] is computed by (2) as the average of the A-scan values within the respective layer.

$$
M V F_{i}(x, y)=\frac{1}{Z_{i}^{2}(x, y)-Z_{i}^{1}(x, y)+1} \sum_{z=Z_{i}^{1}(x, y)}^{Z_{i}^{2}(x, y)} V(x, y, z),
$$

where $\mathrm{V}$ is the $\mathrm{OCT}$ volume (of size $512 \times 512 \times 1024$ voxels), $Z_{i}^{1}(x, y)$ and $Z_{i}^{2}(x, y)$ are the limits of Layer $i(i=\{1,2,3\})$ at coordinates $(x, y)$ and the coordinate system for the OCT data is defined as: $\mathrm{x}$ is the nasal-temporal direction, $\mathrm{y}$ is the superior-inferior direction, and $\mathrm{z}$ is the anterior-posterior (depth) direction [21.

Texture analysis Texture analysis is an image analysis technique used in a wide range of applications with special emphasis on pattern recognition.

In this work we resort to common texture analysis techniques 22 to find whether the structural arrangement of the retina may be different in mice model of $\mathrm{AD}$ in comparison to those of controls. In addition, it may provide further insight into the natural ageing process through the analysis of data from WT animals imaged over time.

In this exploratory approach we make use of the Energy (3) and Contrast (4) statistical properties of the image derived from the gray-level co-occurrence matrix (GLCM). In particular, the number of gray levels of computed fundus reference images was reduced to 16 to limit the size of the GLCM. Furthermore, we split each image into 7 by 7 blocks (of equal size) with an overlap of $10 \%$ and analyze each independently of each other, in different directions and at different scales. On the one hand, with respect to the RNFL, different orientations are expected because fibers converge to the optic disc. On the other hand, when considering the human retina, where the OCT scans are performed centered on the fovea, fibers are radially oriented away from it. The maximum energy and maximum contrast across the combination of directions $\left(0^{\circ}, 45^{\circ}, 90^{\circ}\right.$ and $\left.135^{\circ}\right)$ and scales (1, 2, 3 and 4) was chosen as the value for the block, respectively, for energy and contrast.

$$
\begin{aligned}
E_{\theta}^{\alpha} & =\sum_{i, j} p(i, j)^{2}, \\
C_{\theta}^{\alpha} & =\sum_{i, j}|i-j|^{2} p(i, j),
\end{aligned}
$$

where $p(i, j)$ is the joint probability occurrence of pixel pairs having values $i$ and $j$ in the image and $\alpha$ and $\theta$ are, respectively, the scale and direction. 


\subsection{Classification}

Each eye scan is characterized by a vector of 52 parameters and is labeled as "C", if belonging to the group of controls (WT), and as "D" otherwise (TMM).

Classification is performed using support vector machines (SVM) with radial basis function (RBF) kernel [23. This is a supervised learning algorithm able to infer a function from labeled training data, a set of training examples for which the classification is known beforehand. A model (discriminating function) is thus created and later applied to classify new cases.

As not all the features may be required, the backward elimination algorithm was used to increase the performance (i.e., accuracy in classifying unknown cases) of the system. From the initial set of parameters a subset (of dimension 6) was determined allowing for an increased accuracy. The system accuracy was determined using the $\mathrm{k}$-fold cross-validation procedure, were the cases were split into $k$ sets. $k-1$ sets were used for training and the set left out was then classified using the establish model. The accuracy was determined comparing the obtained classifications into the "C" and "D" classes with the actual ones. The process repeats $k$ times.

The working hypothesis, that OCT embeds information on the status of the CNS allowing to discriminate between controls and $\mathrm{AD}$ cases, will prove to be correct should the system be able to classify eyes, in the WT and TMM groups, with an accuracy over the simple randomization one (equivalent to tossing a coin).

\section{Results}

Two eyes per animal were imaged by the OCT system, at the ages of 4 and 8 months, and the respective OCT volume data exported and processed. The number of eyes and scans performed can be found in table 1. These do not account for the 13 out of $90(14.4 \%)$ scans dropped due to acquisition and segmentation errors.

Table 1. Number of eyes and valid scans performed at the ages of 4 and 8 months. WT - wild type mice (controls), and; TMM - Transgenic mouse model of Alzheimer's disease.

\begin{tabular}{|c|c|c|c|c|}
\hline $\begin{array}{c}\text { Age } \\
\text { (months) }\end{array}$ & \multicolumn{2}{|c|}{ WT } & \multicolumn{2}{c|}{ TMM } \\
\cline { 2 - 5 } & Eyes & Scans & Eyes & Scans \\
\hline 4 & 20 & 20 & 12 & 12 \\
\hline 8 & 26 & 20 & 32 & 25 \\
\hline
\end{tabular}

The number of cases in each class ("C" and "D") for every classification performed was matched every time not to bias results. Cases were randomly selected 


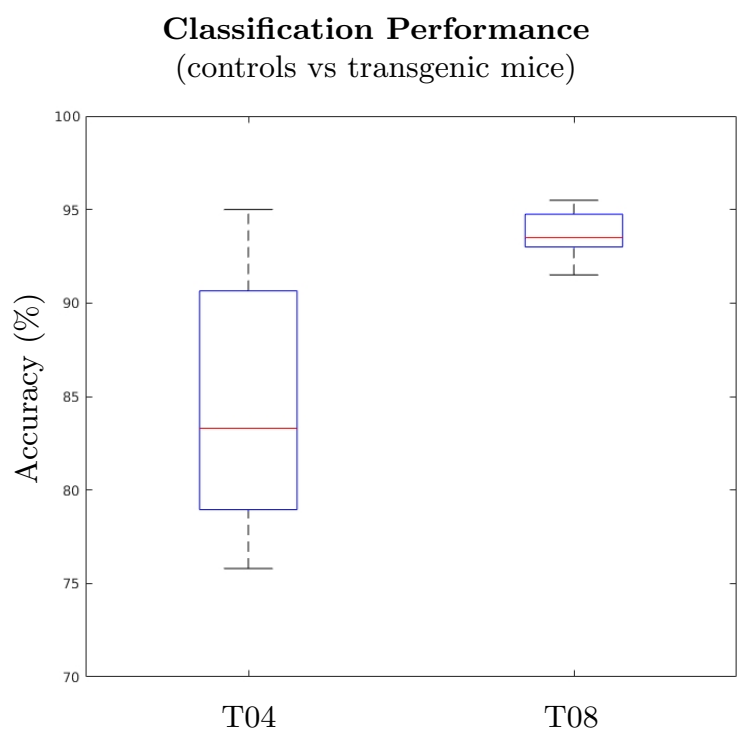

Fig. 2. Classification performance in discriminating wild type mice (WT) from tripletransgenic mouse model (3xTg-AD) (TMM) of Alzheimer's Disease at time points T04 (4 months) and T08 (8 months), respectively at the ages of 4 and 8 months. Minimum/Median/Maximum accuracy values (\%) are of 76/83/95 and 92/94/96, respectively for time points T04 and T08.

and the process repeated multiple times. In consequence, accuracy results are shown as a distribution as opposed to a single value (Fig. 2).

Achieved results strongly suggest that retinas of transgenic mice are actually different from that of healthy controls at the same age. Additionally, results demonstrate that differences do exist at the age of 4 months and that the differences are even clearer at the age of 8 months, as shown by the increase from the minimum $76 \%$ to a minimum of $92 \%$ in classification accuracy (Fig. 2).

Results from the texture analysis can be found in figure 3 At the age of 4 months WT and TMM mice do show similar values for both the energy and contrast at all layers. At the age of 8 months, the contrast is reduced for the TMM group in comparison to that of the WT group. For the energy, differences become clearer. In particular, for Layer 1 the energy distribution is similar but with an increase in the mean value for the TMM group. For Layers 2 and 3, the distributions show that energy values of WT mice do have a much more homogeneous distribution than that of the TMM group. These findings suggest a difference in tissue properties between controls and mouse model of disease which is in agreement with results from the classification using the SVM algorithm. 


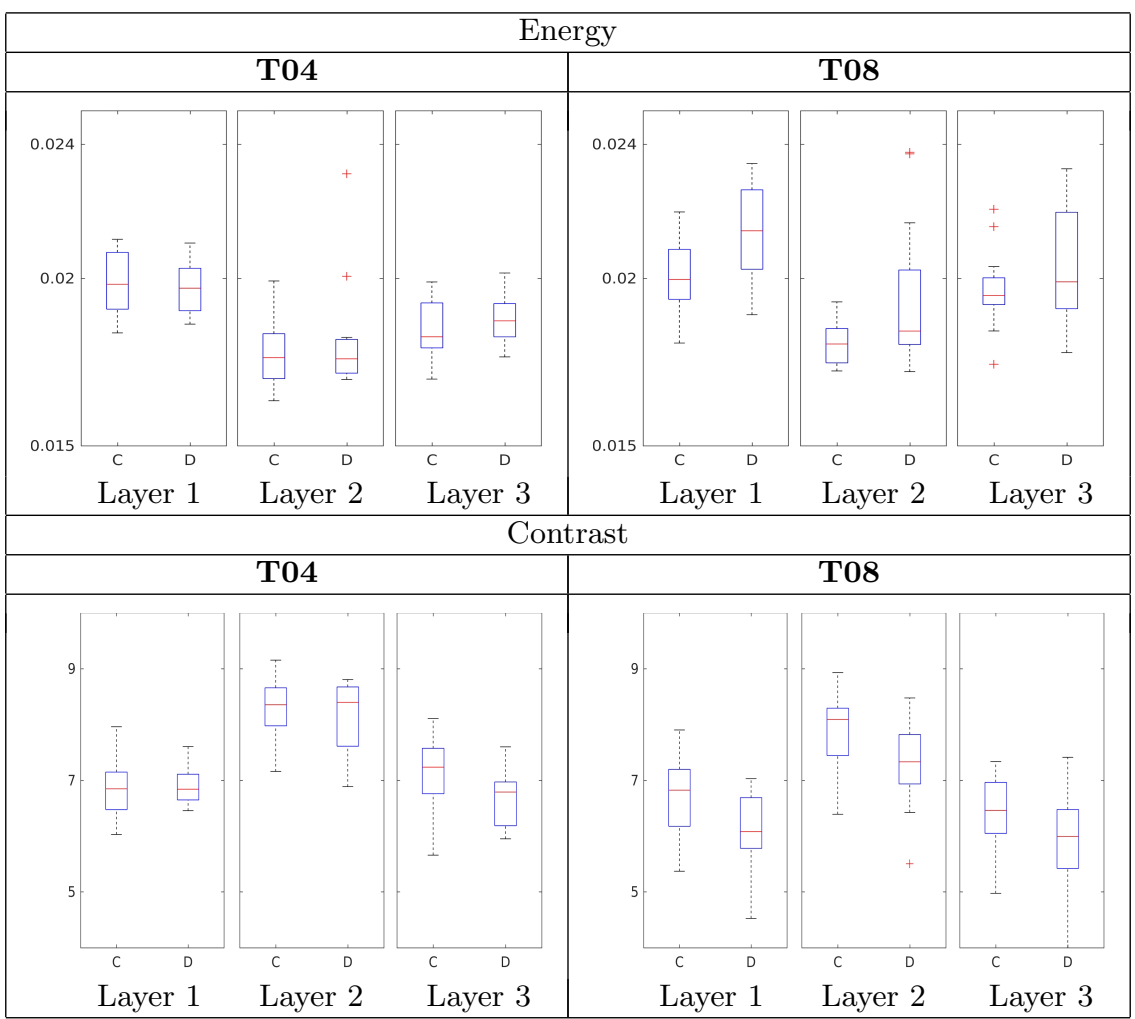

Fig. 3. Texture analysis: energy and contrast from the gray-level co-occurrence matrix (GLCM) of mean value fundus (MVF) images for Layers 1 to 3 and time points T04 and T08, respectively mice at the ages of 4 and 8 months. $\mathrm{C}$ - wild type (WT) mice group; D - transgenic mouse model (TMM) group.

\section{Conclusions}

In this paper we have tested the approach followed previously for the classification of human retinas to find it possible to apply it to OCT scans of mice retinas with success.

Our data show that not only it is possible to use the OCT to get an insight on changes occurring in the retina of mouse model of Alzheimer's disease, but that differences are sufficient to allow to discriminate eyes of controls from that of the transgenic group, with an accuracy in the prediction of over $80 \%$ at the age of 4 months and over $90 \%$ at the age of 8 months (median values).

Finally, found differences in texture of fundus images computed for the layers considered in this work suggest a more heterogeneous organization of the retina in transgenic mice at the age of 8 months, while at the age of 4 months results are similar. 


\section{Acknowledgements}

This study was supported by the Neuroscience Mantero Belard Prize 2015 (Santa Casa da Misericórdia)(MB-1049-2015), by The Portuguese Foundation for Science and Technology (PEst-UID/NEU/04539/2013), by FEDER-COMPETE (POCI-01-0145-FEDER-007440) and Centro 2020 Regional Operational Programme (CENTRO-01-0145-FEDER-000008: BrainHealth 2020).

\section{References}

1. R Bernardes, P Serranho, T Santos, V Gonçalves \& J Cunha-Vaz. Optical coherence tomography: automatic retina classification through support vector machines. European Ophthalmic Review. (6)4:200-3, 2012.

2. M Prince, M Guerchet \& M Prina. The epidemiology and impact of dementia: current state and future trends. World Health Organization, Geneva, 2015.

3. Alzheimer's Association. 2010 Alzheimer's disease facts and figures. Alzheimer's \& dementia, 6(2), 158-194, 2010. doi:10.1016/j.jalz.2010.01.009

4. S Krantic \& A Torriglia. Retina: source of the earliest biomarkers for Alzheimer's disease? Journal of Alzheimer's Disease, 40(2), 237-243, 2014. doi:10.3233/JAD-132105

5. K Strimbu \& JA Tavel. What are biomarkers? Current Opinion in HIV and AIDS, 5(6), 463, 2010. doi:10.1097/COH.0b013e32833ed177

6. MF Cordeiro. Eyeing the brain. Acta Neuropathologica, 132(6), 765-766, 2016. doi:10.1007/s00401-016-1628-z

7. C Duque, C Januário, J Lemos, P Fonseca, A Correia, L Ribeiro, R Bernardes \& A Freire. Optical coherence tomography in LRRK2-associated Parkinson Disease. Neurology, 84(14), Supplement P2.147, 2015.

8. R Bernardes A Correia, OC D'Almeida, S Batista, L Sousa \& M Castelo-Branco. Optical properties of the human retina as a window into systemic and brain diseases. Investigative Ophthalmology \& Visual Science, 55, 3367, 2014.

9. R Sterniczuk, MC Antle, FM LaFerla \& RH Dyck. Characterization of the 3xTg-AD mouse model of Alzheimer's disease: part 2. Behavioral and cognitive changes. Brain research, 1348, 149-155, 2010. doi:10.1016/j.brainres.2010.06.011

10. B Bouma \& G Tearney. Handbook of Optical Coherence Tomography. New York, Marcel Dekker, Inc, 2002.

11. D Huang, EA Swanson, CP Lin, JS Schuman, WG Stinson, W Chang, MR Hee, T Flotte, K Gregory, CA Puliafito \& JG Fujimoto. Optical coherence tomography. Science, 254(5035), 1178-1181, 1991.

12. JG Fujimoto \& W Drexler. "Introduction to optical coherence tomography" in Optical Coherence Tomography: Technology and Applications. W Drexler \& JG Fujimoto, Eds., 1-45, Springer, Berlin, Heidelberg, 2008.

13. MD Abramoff, M Garvin, M Sonka. Retinal Imaging and Image Analysis. IEEE Reviews in Biomedical Engineering, 3, 169-208, 2010. doi:10.1109/RBME.2010.2084567

14. K Kang, X Wu, DZ Chen, M Sonka. Optimal Surface Segmentation in Volumetric Images - A Graph-Theoretic Approach. IEEE Transactions on Pattern Analysis and Machine Intelligence, 28, 119-134, 2006. doi:10.1109/TPAMI.2006.19

15. MK Garvin, MD Abramoff, X Wu, TK Burns, SR Russell, M Sonka. Automated 3-D Intraretinal Layer Segmentation of Macular Spectral-Domain Optical Coherence Tomography Images. IEEE Trans Med. Imaging, 28(9), 1436-47, 2009. doi:10.1109/TMI.2009.2016958 
16. T Hasegawa, HO Ikeda,N Nakano, Y Muraoka, T Tsuruyama, K Okamoto-Furuta, H Kohda \& N Yoshimura. Changes in morphology and visual function over time in mouse models of retinal degeneration: an SD-OCT, histology, and electroretinography study. Jpn J Ophthalmol, 60, 111, 2016. doi:10.1007/s10384-015-0422-0

17. EJ Knott, KG Sheets, Y Zhou, WC Gordon \& NG Bazan. Spatial correlation of mouse photoreceptor-RPE thickness between SD-OCT and histology. Experimental eye research, 92(2), 155-160, 2011. doi:10.1016/j.exer.2010.10.009

18. A Berger, S Cavallero, E Dominguez, P Barbe, M Simonutti, JA Sahel, F Sennlaub, W Raoul, M Paques \& AP Bemelmans. Spectral-Domain Optical Coherence Tomography of the Rodent Eye: Highlighting Layers of the Outer Retina Using Signal Averaging and Comparison with Histology. PLoS ONE, 9(5), e96494, 2014. doi:10.1371/journal.pone.0096494

19. RM Dutescu, QX Li, J Crowston, CL Masters, PN Baird \& JG Culvenor. Amyloid precursor protein processing and retinal pathology in mouse models of Alzheimer's disease. Graefes Arch Clin Exp Ophthalmol, 247, 1213-1221, 2009. doi:10.1007/s00417-009-1060-3

20. P Guimarães, P Rodrigues, C Lobo, S Leal, J Figueira, P Serranho \& R Bernardes. Ocular fundus reference images from optical coherence tomography. Computerized Medical Imaging and Graphics, 38, 381-389, 2014.

21. P Rodrigues, P Guimarães, T Santos, S Simão, T Miranda, P Serranho \& R Bernardes. Two-dimensional segmentation of the retinal vascular network from optical coherence tomography. Journal of Biomedical Optics, 18(12), 126011, 2013.

22. RC Gonzalez, RE Woods. Digital Image Processing (Second Edition). Prentice Hall, New Jersey, 2002.

23. RO Duda, PE Hart \& DG Stork. Pattern Classification. Chichester, UK: WileyInterscience, 2000. 